\title{
EI profesional del siglo XXI al servicio de la sociedad y de las organizaciones
}

\author{
Por Adela d'Alòs-Moner
}

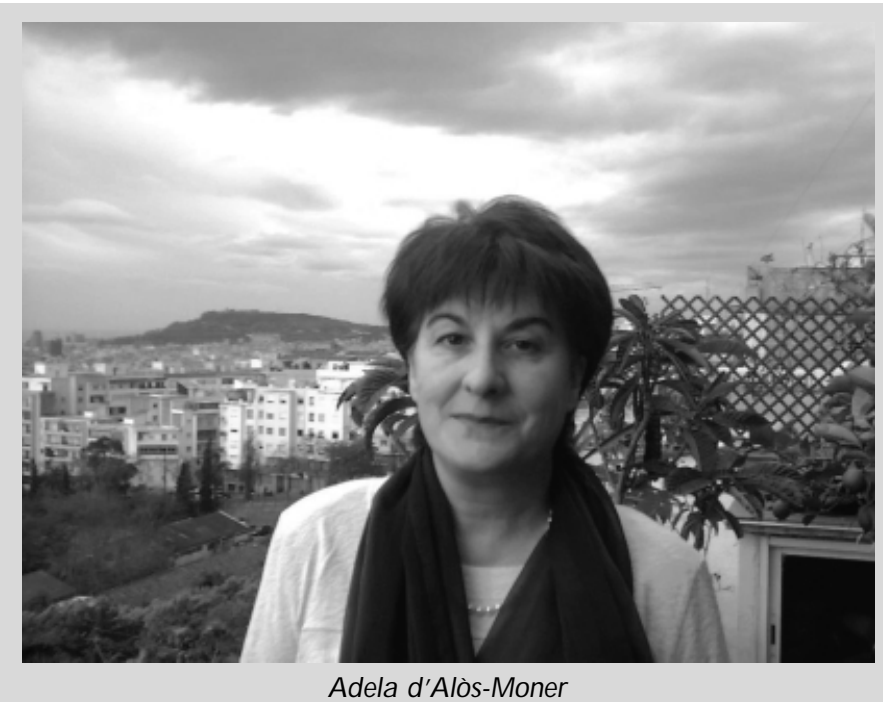

Resumen: El paso de la sociedad industrial a la sociedad de la información y del conocimiento comportará importantes cambios en las organizaciones y un nuevo papel de los servicios de información y de los profesionales. Son necesarias nuevas competencias, gran parte de ellas ligadas a la formación permanente, pero también (y estos aspectos adquieren cada vez mayor importancia) nuevas actitudes y aptitudes. Se demanda un mayor compromiso y ser capaz de aportar valor a la propia organización y a la sociedad en general.

Palabras clave: Sociedad de la información, Sociedad del conocimiento, Papel del profesional, Competencias, Actitudes, Aptitudes.

Title: The professional in the third millennium: a commitment to society and organisations

Abstract: The information and knowledge society that is supplanting the industrial society will produce important changes in organisations and ultimately will require infomation professionals and services to assume new roles. New areas of competence (tied directly to continuing education) will be necessary, as well as new attitudes and aptitudes which will become increasingly more important. Employees will have to contribute to knowledge creation and to offer true value to both the organisation and to the society as a whole.

Keywords: Information society, Knowledge society, Professional roles, Competence, Attitudes, Aptitudes.

Alòs-Moner, Adela d'. "El profesional del siglo XXI al servicio de la sociedad y de las organizaciones". En: El profesional de la información, 2001, diciembre, v. 10, n. 12, pp. 26-29.

\section{¿Cómo serán las organizaciones del siglo XXI? ¿Hacia dónde evolucionarán?}

Muchos investigadores han empezado a describir el nuevo perfil de las organizaciones de este siglo y coinciden en diversos aspectos. En primer lugar hay concordancia en la idea de que éstas, entendidas como una fábrica, con unos procesos definidos y que pueden tardar en modificarse, cambiarán de manera muy rápida. Se perfilan unas instituciones que actúan como los

\section{El profesional de la información debe ser activo en:}

- La garantía de igualdad de acceso a la información de la administración pública.

- La integración y la estabilidad social, resultado de una sociedad más justa y equilibrada.

- El desarrollo de las comunidades económicas locales.

- El crecimiento y la mejora de la eficacia de las organizaciones.

- La calidad global de los servicios. seres vivos, con una necesidad de adaptación constante a las nuevas realidades para poder sobrevivir. Conceptos como "ecología de las organizaciones" los podemos encontrar cada vez con más frecuencia.

\section{«Es evidente que continuarán existiendo grandes empresas pero la virtualidad y la dinámi- ca de internet harán que mu- chas empresas aparezcan y de- saparezcan con una rapidez sorprendente»}

La segunda idea en la que muchos coinciden es que las organizaciones "se harán y desharán" y, como en una película, tendrán un principio y un final, en la que el director busca intérpretes, escenógrafos y músicos que trabajan en equipo durante meses o años. Es evidente que continuarán existiendo grandes empresas (hemos visto en los últimos años una tendencia a su concentración en pocas manos) pero la virtualidad y la dinámica de internet las harán aparecer y desaparecer con una rapidez sorprendente.

Las formas de trabajo también se verán afectadas con esta nueva realidad. Los sindicatos ya han tomado nota de ello y han empezado a organizar secciones especiales para atender las necesidades de un colectivo de profesionales autónomos que cada vez es más im- 


\section{Ovid Technologies}

Las plataformas de Ovid y SilverPlatter le ofrecen ahora más posibilidades de elección

\section{iLo tenemos todo!}

- Ahora más de $\mathbf{3 0 0}$ bases de datos con el contenido más valioso, en todas las áreas temáticas

- Enlazadas con Journals@Ovid: colección de revistas electrónicas con la posibilidad de buscar cada una de las palabras de cada artículo

- Tecnologías de enlace totalmente personalizables

- Nuevas y mejoradas interfaces de búsqueda y recuperación

Ovid Technologies

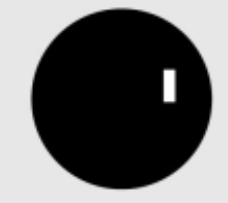

$O \quad V \quad I \quad D$
Madrid Tel : 915678483 Barcelona Tel : 938106563 Email : spain@ovid.com

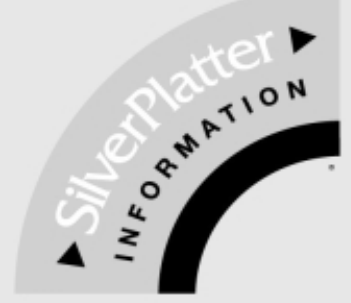


portante. Un tercer aspecto, consecuencia directa de la utilización de las tecnologías de la información y las comunicaciones, es la reducción física de los lugares de trabajo, teniendo en cuenta que en algunas organizaciones se hará desde casa.

Por último, y ésta es seguramente la cuestión que de manera decisiva afectará a los profesionales de la información (bibliotecarios o documentalistas), es el papel cada vez más importante que tiene la información y el conocimiento en las organizaciones.

Estos últimos años se han caracterizado por un importante cambio económico, social, político y cultural que se traduce en el paso de la sociedad industrial a otra basada en la información y el conocimiento. Las previsiones indican que estamos en un momento de transición y que será a partir de 2004 cuando la sociedad de la información supere a la industrial en la mayoría de los países occidentales. Más del $60 \%$ de la población mundial (hay que recordarlo) queda fuera de esta realidad y lo estará durante muchos años.

\section{«Estamos entrando de lleno en una sociedad marcada por los avances tecnológicos y por una nueva cultura de las orga- nizaciones en la que la informa- ción y el conocimiento tienen cada vez más importancia»}

Podemos decir que estamos entrando de lleno en una sociedad incipiente marcada por los avances tecnológicos y por una nueva cultura informacional de las organizaciones; en una nueva era en la que la riqueza se encuentra, cada vez menos, en los objetos tangibles y cada vez más en la información y el conocimiento.

\section{EI nuevo rol de los servicios de información y de los profesionales}

En este contexto inédito, en el que la información tiene un papel tan decisivo, se abren unas enormes posibilidades para el profesional de la información. Sin embargo, para aprovecharlas es necesario que se comprometa con la organización en la cual trabaja y con la sociedad en general. Todo ello comporta, en definitiva, tener un papel activo en la garantía de una sociedad más justa y en el crecimiento económico del país.

Ahora ya no es suficiente gestionar de manera más o menos eficaz un servicio de información (seleccionar, describir y difundir la información) sino que hay que ser capaz de situarse junto al motor de la organización, ser un elemento central que incide en su crecimiento.
En todos los ámbitos laborales afloran nuevas necesidades, y lo que más se demanda son actitudes de receptividad ante los cambios, saber actuar como un aliado en la dirección de la organización y tener capacidad para afrontar los retos que se presentan y los compromisos asumidos de manera innovadora. Los profesionales de la información no sólo forman parte de esta ola, sino que deben saber situarse en su cresta como elementos centrales, dinamizadores del cambio y de la nueva sociedad.

\section{«Ahora ya no es suficiente ges- tionar de manera más o menos eficaz un servicio de informa- ción, sino que hay que ser ca- paz de situarse en el corazón de la organización, ser un ele- mento central que incide en su crecimiento»}

En este marco, los servicios de información dejan de ser un departamento más de las organizaciones para pasar a situarse en su mismo corazón. Ya no se trata de gestionar de manera más o menos acertada un departamento dentro de una empresa u organización, sino de ver cómo participar en su transformación y adecuación a la nueva realidad.

\section{Conocimientos, aptitudes y actitudes}

Se demandan nuevos conocimientos, así como unas actitudes y aptitudes determinadas. El reconocido analista de organizaciones Peter Drucker insiste en señalar que este final o inicio de siglo será recordado, más que por avances tecnológicos, por los profundos cambios en las profesiones. Por primera vez, señala, todos estaremos obligados a lo largo de nuestra vida a tomar diferentes opciones de tipo laboral. Cada vez habrá una mayor distancia entre lo que se ha estudiado y los trabajos que se realizan. Si esto es cierto para la mayoría de las actividades, es innegable para los profesionales de la información. Si hace unos años ésta se encontraba ligada directamente a un título, hoy es únicamente un punto de partida, y tenerlo ya no implica ser un profesional. La profesionalidad debe expresarse con un compromiso social y colectivo, encontrándose estrechamente relacionada con aspectos como el aprendizaje permanente.

El nuevo profesional de la información necesita de unos nuevos conocimientos pero también, y esto es cada vez más importante, de unas determinadas actitudes y aptitudes. En primer lugar, debe tener visión de futuro, ser capaz de prever y anticiparse a escenarios que están aún por venir, saber enfrentarse a los continuos cambios. Es más, debe conocer la forma de actuar pa- 
ra convertirse en el catalizador de dichas transformaciones y ayudar a la organización a adaptarse a las nuevas realidades.

\section{«Si hace unos años la profe- sión se encontraba ligada di- rectamente a un título, hoy es únicamente un punto de parti- da, y tenerlo ya no implica ser un profesional»}

Desgraciadamente, una herramienta importante como internet ha tenido un papel perverso ya que muchos han considerado que son unos buenos profesionales por el simple hecho de saber moverse con agilidad por la Red o dominar los lenguajes de edición electrónica. Internet ha desvirtuado a menudo el papel del nuevo profesional, que debe centrarse en ver cómo puede aportar valor a la organización, a sus usuarios, clientes o ciudadanos. Para poder añadir valor es indispensable conocer las necesidades y expectativas cambiantes de los usuarios, en un entorno también cambiante. En general, el profesional de la información debe centrarse en los siguientes puntos:

a. Unos conocimientos actualizados permanentemente para asegurar la puesta al día del título, que deberán incluir aspectos tan diversos como:

- Saber cómo funcionan las organizaciones y conocer la forma en la que se toman las decisiones.

- Transmitir la información de manera personalizada en función de quien sea su destinatario.

b. Unas aptitudes para:

-El trabajo en equipo.

—La comunicación y las relaciones públicas.

- Tener un rol de liderazgo en la organización.

- Mostrar cómo el servicio aporta valor a la organización y genera beneficios. Para plasmarlo con dos ejemplos concretos, éstos pueden consistir en ganancias económicas (en la empresa) o en integración social y estabilidad política del entorno en el que actúa (en una biblioteca pública).

c. Unas actitudes ligadas a:

-Un trabajo que se identifica con la misión y los valores corporativos.

—La preocupación por la mejora continua.

—Identificación y anticipación a las necesidades y expectativas.

- La conciencia de la necesidad de aportar valor a la organización.

—Asumir y saber compartir responsabilidades.

- Implicarse activamente en el entorno profesional con participación en asociaciones, jornadas, publicaciones, etc.

\section{«Desgraciadamente, una herra- mienta importante como inter- net ha tenido un papel perverso ya que muchos han considera- do que son unos buenos profe- sionales por el simple hecho de saber moverse con agilidad por la Red o dominar los lenguajes de edición electrónica»}

Se abren enormes posibilidades para los profesionales de la información. El reto hoy es saberlas aprovechar teniendo un mayor papel social como colectivo, con una imagen más innovadora y comprometida.

Adela d'Alòs-Moner, DOC6, Consultores en recursos de información.

aalos@doc6.es

http://www.doc6.es

\section{Próximos números monográficos \\ Enero 2002 Intranet y documentación \\ Marzo 2002 Automatización de bibliotecas \\ Mayo 2002 Externalización y subcontratación de servicios}

Los interesados pueden remitir notas, artículos, propuestas, publicidad, comentarios, etc., sobre estos temas a:

epi@ sarenet.es 